\title{
DYNAMIC REYNOLDS EQUATION FOR MICROPOLAR FLUID LUBRICATION OF POROUS SLIDER BEARINGS
}

Neminath Bhujappa Naduvinamani

Department of Mathematics, Gulbarga University Gulbarga-585106, India., naduvinamaninb@yahoo.co.in

Gudadappagouda Basanagouda Marali

Department of Mathematics, B V B college of Engineering and Technology, Vidyanagar, Hubli-580031, India.

Follow this and additional works at: https://jmstt.ntou.edu.tw/journal

Part of the Electrical and Computer Engineering Commons

\section{Recommended Citation}

Naduvinamani, Neminath Bhujappa and Marali, Gudadappagouda Basanagouda (2008) "DYNAMIC REYNOLDS EQUATION FOR MICROPOLAR FLUID LUBRICATION OF POROUS SLIDER BEARINGS," Journal of Marine Science and Technology. Vol. 16: Iss. 3, Article 3.

DOI: $10.51400 / 2709-6998.2002$

Available at: https://jmstt.ntou.edu.tw/journal/vol16/iss3/3

This Research Article is brought to you for free and open access by Journal of Marine Science and Technology. It has been accepted for inclusion in Journal of Marine Science and Technology by an authorized editor of Journal of Marine Science and Technology. 


\section{DYNAMIC REYNOLDS EQUATION FOR MICROPOLAR FLUID LUBRICATION OF POROUS SLIDER BEARINGS}

\section{Acknowledgements}

The authors sincerely acknowledge the financial assistance by the UGC, New Delhi, India under the major research project No.F.No.31-84/2005 (SR) and one of the authors (GBM) thankful to Dr. Ashok S. Shettar, Principal, B.V.B. College of Engineering and Technology, Hubli-31 for his encouragements. 


\title{
DYNAMIC REYNOLDS EQUATION FOR MICROPOLAR FLUID LUBRICATION OF POROUS SLIDER BEARINGS
}

\author{
Neminath Bhujappa Naduvinamani* and Gudadappagouda Basanagouda Marali**
}

Key words: dynamic stiffness, dynamic damping coefficient, micropolar fluids, porous, slider bearings.

\begin{abstract}
In this paper an attempt has been made to study the rheological effects of micropolar fluid lubricants on the steady state and dynamic behavior of porous slider bearings by considering the squeezing action. The general modified Reynolds-type equation of porous sliding-squeezing surfaces with micropolar fluids is derived for the assessment of dynamic characteristics of bearings with general film thickness. The detailed analysis is presented for the plane inclined porous slider bearings by using perturbation method. Two Reynolds-type equations corresponding to steady performance and perturbed characteristics are obtained. The closed form solution of these equations is obtained. The numerical computations of the results show that, there exists a critical value for profile parameter at which the steady-state load and dynamic stiffness coefficient attains maximum. Further the micropolar fluids provide improved characteristics for both steady-state and the dynamic stiffness and dynamic damping characteristics. It is found that the maximum steady load carrying capacity is function of coupling parameter and the permeability parameter and is achieved at smaller values of profile parameter for larger values of the coupling parameter.
\end{abstract}

\section{INTRODUCTION}

Porous bearings have the features of simple structure and low cost. Porous bearings are used where non-porous bearings are impracticable owing to lack of space or inaccessibility for lubrication. The application of porous bearings in mounting horsepower motors include vacuum cleaners, coffee grinders, hair driers, saving machines, sewing machines, water pumps, record players, tape recorders, generators and distributors. Several researchers $[9,10,15,16,18]$ have analyzed the porous slider bearings by using Darcy's equation to model the flow of Newtonian lubricant in the porous matrix. All these studies

Paper submitted 04/13/07; accepted 06/04/07. Author for correspondence: Neminath Bhujappa Naduvinamani (e-mail:naduvinamaninb@yahoo.co.in). *Department of Mathematics, Gulbarga University Gulbarga-585106, India.

**Department of Mathematics, B V B college of Engineering and Technology, Vidyanagar, Hubli-580031, India. assume the lubricant to be a Newtonian fluid.

Micropolar fluids contain a suspension of particles with individual motion. These particles support stress and body moments [5, 6] and are subjected to spin inertia and hence microscopic effects generated by the local structure and micro motions of fluid elements are exhibited. The theory of micropolar fluids was first presented by Eringen [5] as a particular case of microfluids, which includes the effects of local rotatary inertia, couple stresses and inertial spin. This model is applicable for the study of flow properties of non-Newtonian fluids such as polymers, paints, lubricants, suspended fluids, blood etc The study of micropolar fluids has received considerable attention due to applications in a number of processes that occur in industries such as the extrusion of polymer fluids, solidification of liquid crystals, cooling of metallic plate in a bath, exotic lubricants and colloidal and suspension solution. In the study of all these problems the classical Navier-Stokes theory is inadequate. The micropolar fluid theory is a subclass of microfluid theory and is obtained by imposing the skew symmetric properties of the gyration tensor in addition to the condition of micro-isotropy. Several investigators used the micropolar fluid theory for the study of several bearing systems such as slider bearings [4, 14], journal bearings [7, 8, 20], squeeze film bearings [1, 2, 3, 13] and porous bearings [19, 21, 22] and have found some advantages of micropolar fluids over the Newtonian lubricants such as increased load carrying capacity and delayed time of approach for squeeze film bearings. The generalized Reynolds equation for micropolar lubricants is derived by Sukla and Isa [17] for one dimensional slider bearings by neglecting the squeezing action of the bearing. However no attempt has been made to study the static and dynamic characteristics of porous inclined slider bearings with micropolar fluids by including the squeezing action of the bearing surfaces. The objective of this paper is to derive the generalized dynamic Reynolds-type equation of porous sliding-squeezing surfaces with micropolar fluids, as it is necessary for the study of dynamic characteristics of lubricating system. As an illustration detailed analysis is presented for infinitely wide porous plane inclined slider bearings.

\section{DERIVATION}

The following assumptions in addition to the usual 
assumptions of lubrication theory for thin films [12] are used in the present analysis

1. The micropolar fluid flow in the film region is laminar.

2. Inertial forces are negligible compared to viscous terms.

3. The pressure is independent of the $y$-coordinate and $y$-derivatives of the velocity components and micro rotational velocity components dominate.

4. The porous layer thickness $H$ to be small.

5. The body forces and body couples are assumed to be absent and the characteristic coefficients across the film of the micropolar fluid are constant.

The basic equations governing the flow of micropolar lubricants [6] under the usual assumptions of lubrication theory for thin films are [12]

$$
\begin{gathered}
\left(\mu+\frac{\chi}{2}\right) \frac{\partial^{2} u}{\partial y^{2}}+\chi \frac{\partial v_{3}}{\partial y}-\frac{\partial p}{\partial x}=0 \\
\frac{\partial p}{\partial y}=0 \\
\left(\mu+\frac{\chi}{2}\right) \frac{\partial^{2} w}{\partial y^{2}}-\chi \frac{\partial v_{1}}{\partial y}-\frac{\partial p}{\partial z}=0 \\
\gamma \frac{\partial^{2} v_{1}}{\partial y^{2}}-2 \chi v_{1}+\chi \frac{\partial w}{\partial y}=0 \\
\gamma \frac{\partial^{2} v_{3}}{\partial y^{2}}-2 \chi v_{3}-\chi \frac{\partial u}{\partial y}=0 \\
\frac{\partial u}{\partial x}+\frac{\partial v}{\partial y}+\frac{\partial w}{\partial z}=0
\end{gathered}
$$

where $(u, v, w)$ are velocity components along $x, y, z$-directions and $\left(v_{1}, v_{2}, v_{3}\right)$ are micro rotational velocity components, $\chi$ and $\gamma$ are additional viscosity coefficients for micropolar fluids.

The physical configuration of the problem under consideration is shown in the Fig. 1. It consists of porous slider bearing with sliding velocity $U$ including the effect of squeezing action $\frac{\partial h}{\partial t}, \mathrm{~h}_{1}(\mathrm{t})$ is the inlet film thickness and outlet film thickness is $\mathrm{h}_{0}(\mathrm{t})$.

The relevant boundary conditions for the velocity and micro rotational velocity components are

(i) at the upper surface $(y=h)$

$$
\begin{gathered}
u=w=0, v=\frac{\partial h}{\partial t} \\
v_{1}=\mathrm{v}_{3}=0
\end{gathered}
$$

(ii) at the lower surface $(y=0)$

$$
\begin{gathered}
u=U, v=v^{*} \\
w=0 \\
v_{1}=v_{3}=0
\end{gathered}
$$

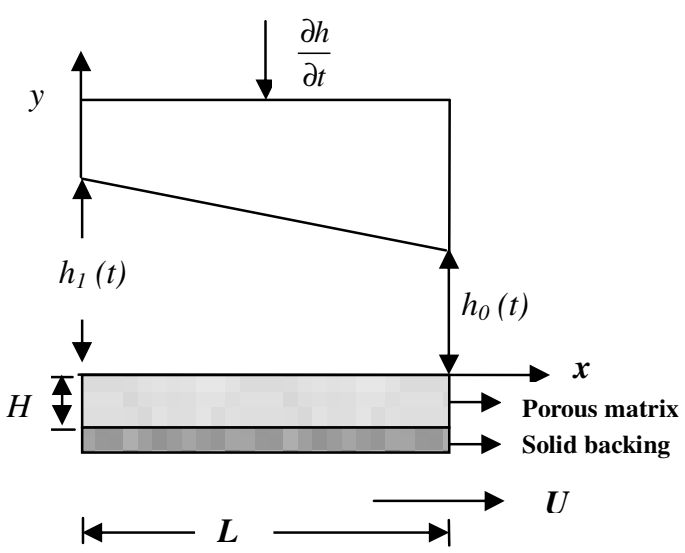

Fig. 1. Physical geometry of porous inclined slider bearing.

The solution of the Eqs. (1), (3), (4) and (5) subject to the corresponding boundary conditions given in Eq. (7) and Eq. (8) are obtained in the form:

$$
\begin{gathered}
u=\frac{1}{\mu}\left(\frac{y^{2}}{2} \frac{\partial p}{\partial x}+C_{11} y\right) \\
-\frac{2 N^{2}}{m}\left[C_{21} \sinh (m y)+C_{31} \cosh (m y)\right]+C_{41} \\
w=\frac{1}{\mu}\left(\frac{y^{2}}{2} \frac{\partial p}{\partial z}+C_{12} y\right) \\
-\frac{2 N^{2}}{m}\left[C_{22} \sinh (m y)+C_{32} \cosh (m y)\right]+C_{42} \\
v_{1}=\frac{1}{2 \mu}\left[y \frac{\partial p}{\partial z}+C_{12}\right]+\left[C_{22} \cosh (m y)+C_{32} \sinh (m y)\right] \\
v_{3}=\left[C_{21} \cosh (m y)+C_{31} \sinh (m y)\right]-\frac{1}{2 \mu}\left(y \frac{\partial p}{\partial x}+C_{11}\right)
\end{gathered}
$$

where

$$
\begin{aligned}
C_{11} & =2 \mu C_{21} \\
C_{21} & =\frac{C_{31} \sinh (m h)-\frac{h}{2 \mu} \frac{\partial p}{\partial x}}{1-\cosh (m h)} \\
C_{31} & =\frac{1}{\mu}\left(\frac{-U}{2}\{1-\cosh (m h)\}\right) \\
+ & \frac{h}{2 \mu} \frac{\partial p}{\partial x}\left(\frac{h}{2}\{\cosh (m h)-1\}+h-\frac{N^{2}}{m} \sinh (m h)\right) \cdot \frac{1}{C_{5}} \\
C_{41} & =U+\frac{2 N^{2}}{m} C_{31} \\
C_{5} & =\frac{h}{\mu}\left[\sinh (m h)-\frac{2 N^{2}}{m h}\{\cosh (m h)-1\}\right]
\end{aligned}
$$


in which $m=\frac{N}{l}, \quad N=\left[\frac{\chi}{\chi+2 \mu}\right]^{1 / 2}, l=\left(\frac{\gamma}{4 \mu}\right)^{1 / 2}$

where $N$ is a non-dimensional parameter called coupling number for it characterizes the coupling of linear and angular momentum equations, when $N$ is identically zero, the equations of linear and angular momentum decoupled and the equation of linear momentum reduces to classical Navier-Stokes equation. The parameter $l$ is called the characteristic length for it characterizes the interaction between the micropolar fluid and the film gap. The parameter $l$ is of dimension length and can be identified as a size of the lubricant molecule. In the limiting case of $l \rightarrow 0$, the microstructure becomes negligible.

Integrating the equation of continuity (6) with respect to $y$ over the film thickness, gives

$$
\int_{y=0}^{h} \frac{\partial v}{\partial y} d y=-\int_{y=0}^{h}\left(\frac{\partial u}{\partial x}+\frac{\partial w}{\partial z}\right) d y
$$

By replacing the velocity components $\mathrm{u}$ and $\mathrm{w}$ with their expressions given in Eq. (9) and Eq. (10) and also using the corresponding boundary conditions given in Eq. (7) and Eq. (8), Eq. (13) gives the dynamic Reynolds type equation for micropolar fluid in the form

$$
\begin{gathered}
\frac{\partial}{\partial x}\left[f(N, l, h) \frac{\partial p}{\partial x}\right]+\frac{\partial}{\partial z}\left[f(N, l, h) \frac{\partial p}{\partial z}\right]= \\
12 \mu \frac{\partial h}{\partial t}+6 \mu U \frac{\partial h}{\partial x}-12 \mu\left(v^{*}\right)_{y=0}
\end{gathered}
$$

where

$$
f(N, l, h)=h^{3}+12 l^{2} h-6 N^{2} l h^{2} \operatorname{coth}\left(\frac{N h}{2 l}\right)
$$

The flow of micropolar lubricants in the porous matrix is governed by modified Darcy's law

$$
q=-\frac{\phi}{\mu+\chi} \nabla p^{*}
$$

where $q=\left(u^{*}, v^{*}, w^{*}\right)$ is the modified Darcy's velocity vector, $\phi$ is the permeability of the porous matrix and $p^{*}$ is the pressure in the porous matrix. Due to continuity of the fluid in the porous matrix, the pressure $p^{*}$ satisfies the Laplace equation.

$$
\frac{\partial^{2} p^{*}}{\partial x^{2}}+\frac{\partial^{2} p^{*}}{\partial y^{2}}+\frac{\partial^{2} p^{*}}{\partial z^{2}}=0
$$

Integrating with respect to y over the porous layer thickness $H$ and using the boundary condition of the solid backing $\left(\frac{\partial p^{*}}{\partial y}=0\right)$ at $y=-H$, we obtain

$$
\left(\frac{\partial p^{*}}{\partial y}\right)_{y=0}=-\int_{-H}^{0}\left(\frac{\partial^{2} p^{*}}{\partial x^{2}}+\frac{\partial^{2} p^{*}}{\partial z^{2}}\right) d y
$$

Assuming that the porous layer thickness $H$ to be small and using the continuity condition of pressure $\left(p=p^{*}\right)$ at the porous interface $(y=0)$. Equation $(18)$ reduces to

$$
\left(\frac{\partial p^{*}}{\partial y}\right)_{y=0}=-H\left(\frac{\partial^{2} p}{\partial x^{2}}+\frac{\partial^{2} p}{\partial z^{2}}\right)
$$

then the velocity component $v^{*}$ at the interface $(y=0)$ is given by

$$
\left(v^{*}\right)_{y=0}=\frac{\phi H}{\mu+\chi}\left(\frac{\partial^{2} p}{\partial x^{2}}+\frac{\partial^{2} p}{\partial z^{2}}\right)
$$

Substituting this in Eq. (14), the dynamic Reynolds equation is obtained in the form

$$
\begin{aligned}
& \frac{\partial}{\partial x}\left[\left(f(N, l, h)+\frac{12 \mu \phi \mathrm{H}}{\mu+\chi}\right) \frac{\partial p}{\partial x}\right]+\frac{\partial}{\partial z}\left[\left(f(N, l, h)+\frac{12 \mu \phi \mathrm{H}}{\mu+\chi}\right) \frac{\partial p}{\partial z}\right] \\
& \quad=12 \mu \frac{\partial h}{\partial t}+6 \mu U \frac{\partial h}{\partial x}
\end{aligned}
$$

where $f(N, l, h)=h^{3}+12 l^{2} h-6 N^{2} l h^{2} \operatorname{coth}\left(\frac{N h}{2 l}\right)$.

In the particular case of $U=0$ and $\phi=0$, the Eq. (21) reduces to pure squeeze film lubrication of bearings with micropolar fluids [2]. The case of plane inclined slider bearings studied by Ramanaiah and Dubey [14] can be recovered when $h=h(x, y)$. In the limiting case of $l \rightarrow 0$ the Eq. (14) reduces to the corresponding Newtonian case [12].

\section{POROUS PLANE INCLINED SLIDER BEARING}

A schematic diagram of the porous plane inclined slider bearing with squeezing action is shown in the Fig. 1. To study the static and dynamic characteristics of the porous plane inclined slider bearing, the film thickness is separated into two parts: the minimum film thickness $h_{m}(t)$ and the slider profile function $h_{s}(x)$

$$
h(x, t)=h_{\mathrm{m}}(t)+h_{s}(x)=h_{\mathrm{m}}(t)+a\left(1-\frac{x}{L}\right)
$$

where $a=h_{1}(t)-h_{0}(t)$.

Introducing thé non-dimensional quantities:

$$
\begin{aligned}
& \bar{h}=\frac{h}{h_{m 0}}, \bar{l}=\frac{l}{h_{m 0}}, \bar{p}=\frac{p h_{m 0}^{2}}{\mu U L}, \bar{t}=\frac{U t}{L}, \\
& \bar{x}=\frac{x}{L}, \bar{z}=\frac{z}{B}, \psi=\frac{\phi H}{h_{m_{0}}^{3}}
\end{aligned}
$$


into the dynamic Reynolds-type equation (21) gives

$$
\begin{aligned}
& \frac{\partial}{\partial \bar{x}}\left[\left(\bar{f}(N, \bar{l}, \bar{h})+12 \psi\left\{\frac{1-N^{2}}{1+N^{2}}\right\}\right) \frac{\partial \bar{p}}{\partial \bar{x}}\right] \\
& +\frac{1}{\delta^{2}} \frac{\partial}{\partial \bar{z}}\left[\left(\bar{f}(N, \bar{l}, \bar{h})+12 \psi\left\{\frac{1-N^{2}}{1+N^{2}}\right\}\right) \frac{\partial \bar{p}}{\partial \bar{z}}\right] \\
& =12 \frac{d \bar{h}_{m}}{d \bar{t}}+6 \frac{d \bar{h}_{s}}{d \bar{x}}
\end{aligned}
$$

where

$$
\bar{f}(N, \bar{l}, \bar{h})=\bar{h}^{3}+12 \bar{h} \bar{l}^{2}-6 N \overline{l h}^{2} \operatorname{coth}\left(\frac{N \bar{h}}{2 \bar{l}}\right)
$$

where $\bar{h}(\bar{x}, \bar{t})=\bar{h}_{m}(\bar{t})+\bar{h}_{s}(\bar{x})=\bar{h}_{m}(\bar{t})+\alpha(1-\bar{x})$ with $\alpha=\frac{a}{h_{m 0}}$.

For infinitely wide porous plane inclined slider bearing Eq. (23) reduces to

$$
\begin{aligned}
& \frac{\partial}{\partial \bar{x}}\left[\left(\bar{f}(N, \bar{l}, \bar{h})+12 \psi\left\{\frac{1-N^{2}}{1+N^{2}}\right\}\right) \frac{\partial \bar{p}}{\partial \bar{x}}\right] \\
& =12 \frac{d \bar{h}_{m}}{d \bar{t}}+6 \frac{d \bar{h}_{s}}{d \bar{x}}
\end{aligned}
$$

The steady and dynamic characteristics of the porous slider bearings are obtained by using the perturbations in $h_{m 0}$. The minimum film thickness and the local film pressure are assumed to be of the form

$$
\bar{h}_{m}=1+\varepsilon e^{i \bar{t}}, \bar{p}=\bar{p}_{0}+\bar{p}_{1} \varepsilon e^{i \bar{t}}
$$

where $\varepsilon$ is the perturbation amplitude and is assumed to be small and $i=\sqrt{-1}$.

Substituting into the dynamic Reynolds-type equation (25) and neglecting the higher order terms of $\varepsilon$, the two Reynolds-type equations corresponding to both steady-state pressure and the perturbed film pressure are obtained in the form

$$
\begin{aligned}
& \frac{\partial}{\partial \bar{x}}\left[\left(\bar{f}_{0}\left(N, \bar{l}, \bar{h}_{s}\right)+12 \psi\left\{\frac{1-N^{2}}{1+N^{2}}\right\}\right) \frac{\partial \bar{p}_{0}}{\partial \bar{x}}\right]=6 \frac{d \bar{h}_{s}}{d \bar{x}} \\
& \frac{\partial}{\partial \bar{x}}\left[\left(\bar{f}_{0}\left(N, \bar{l}, \bar{h}_{s}\right)+12 \psi\left\{\frac{1-N^{2}}{1+N^{2}}\right\}\right) \frac{\partial \bar{p}_{1}}{\partial \bar{x}}+\bar{f}_{1}\left(N, \bar{l}, \bar{h}_{s}\right) \frac{\partial \bar{p}_{0}}{\partial \bar{x}}\right] \\
& =12 i
\end{aligned}
$$

where

$$
\begin{gathered}
\bar{f}_{0}\left(N, \bar{l}, \bar{h}_{s}\right)=\left(1+\bar{h}_{s}\right)^{3}+12 \bar{l}^{2}\left(1+\bar{h}_{s}\right) \\
-6 N \bar{l}\left(1+\bar{h}_{s}\right)^{2} \operatorname{coth}\left(\frac{N\left(1+\bar{h}_{s}\right)}{2 \bar{l}}\right)
\end{gathered}
$$

$$
\begin{aligned}
& \bar{f}_{1}\left(N, \bar{l}, \bar{h}_{s}\right)=3\left(1+\bar{h}_{s}\right)^{2}+12 \bar{l}^{2} \\
& -12 N \bar{l}\left(1+\bar{h}_{s}\right) \operatorname{coth}\left(\frac{N\left(1+\bar{h}_{s}\right)}{2 \bar{l}}\right) \\
& -3 N^{2}\left(1+\bar{h}_{s}\right)^{2} \operatorname{csch}^{2}\left(\frac{N\left(1+\bar{h}_{s}\right)}{2 \bar{l}}\right)
\end{aligned}
$$

The boundary conditions for the steady and perturbed film pressure are

$$
\begin{array}{ll}
\bar{p}_{0}=0 & \text { at } \bar{x}=0, \bar{x}=1 \\
\bar{p}_{1}=0 & \text { at } \bar{x}=0, \bar{x}=1
\end{array}
$$

Integrating Eq. (27) with respect to $\bar{x}$ we obtain

$$
\frac{\partial \bar{p}_{0}}{\partial \bar{x}}=\frac{\sigma\left(\bar{h}_{s}-h_{s_{1}}\right)}{G_{1}}
$$

where $h_{s_{1}}$ is the film thickness at which $\bar{p}_{0}$ is maximum and

$$
G_{1}=\bar{f}_{0}\left(N, \bar{l}, \bar{h}_{s}\right)+12 \psi\left\{\frac{1-N^{2}}{1+N^{2}}\right\}
$$

The solution of (33) subject to the boundary conditions (31) is

$$
\bar{p}_{0}=\int_{0}^{\bar{x}} \frac{6\left(\bar{h}_{s}-h_{s_{1}}\right)}{G_{1}} d \bar{x}
$$

where $h_{s_{1}}=\frac{\int_{0}^{1} \frac{\bar{h}_{s}}{G_{1}} d \bar{x}}{\int_{0}^{1} \frac{1}{G_{1}} d \bar{x}}$

The solution of Eq. (28) subject to the boundary conditions (32) gives the perturbed film pressure in terms of the real and imaginary parts as

$$
\bar{p}_{1}=\bar{p}_{11}+\overline{i p}_{12}
$$

where

$$
\begin{gathered}
\bar{p}_{11}=\int_{0}^{\bar{x}}\left[\frac{G_{1} d_{31}-6 \bar{f}_{1}\left(\bar{h}_{s}-h_{s 1}\right)}{G_{1}^{2}}\right] d \bar{x} \\
\bar{p}_{12}=\int_{0}^{\bar{x}}\left[\frac{\left(12 \bar{x}-d_{32}\right)}{G_{1}}\right] d \bar{x}
\end{gathered}
$$

in these $\quad d_{31}=\frac{\int_{0}^{1} \frac{6 \bar{f}_{1}\left(\bar{h}_{s}-h_{s 1}\right)}{G_{1}^{2}} d \bar{x}}{\int_{0}^{1} \frac{1}{G_{1}} d \bar{x}}, \quad d_{32}=\frac{\int_{0}^{1} \frac{12 \bar{x}}{G_{1}} d \bar{x}}{\int_{0}^{1} \frac{1}{G_{1}} d \bar{x}}$

The steady-state load capacity $W_{s}$ and perturbed film force $W_{d}$ are evaluated by integrating the steady-state film pressure 
and perturbed film pressure respectively over the film region. In terms of non-dimensional quantities these are obtained in the form

$$
\begin{aligned}
& \bar{W}_{S}=\int_{\bar{x}=0}^{\bar{x}=1} \bar{p}_{0} d \bar{x}, \\
& \bar{W}_{d}=\int_{\bar{x}=0}^{\bar{x}=1} \bar{p}_{1} d \bar{x}
\end{aligned}
$$

From the linear theory, the resulting dynamic film force can be expressed in terms of linearized spring and damping coefficients.

$$
W_{d} \varepsilon e^{i \bar{t}}=-S_{d} h_{m 0} \varepsilon e^{i \bar{t}}-C_{d} \frac{d}{d \bar{t}}\left(h_{m 0} \varepsilon e^{i \bar{t}}\right) .
$$

which in non-dimensional form

$$
\bar{W}_{d}=-\bar{S}_{d}-i \bar{C}_{d}
$$

The non-dimensional dynamic stiffness coefficient $\bar{S}_{d}$ and the dynamic damping coefficient $\bar{C}_{d}$ are obtained by equating the real and imaginary parts of $\bar{W}_{d}$ respectively as

$$
\begin{aligned}
& \bar{S}_{d}=-\operatorname{Re}\left(\bar{W}_{d}\right) \approx-\int_{\bar{x}=0}^{1} \bar{p}_{11} d \bar{x} \\
& \bar{C}_{d}=-\operatorname{Im}\left(\bar{W}_{d}\right) \approx-\int_{\bar{x}=0}^{1} \bar{p}_{12} d \bar{x}
\end{aligned}
$$

\section{RESULTS AND DISCUSSION}

To study the lubricating effectiveness of the micropolar fluids, the two non-dimensional parameters are of interest. The coupling number $N=\left(\frac{\chi}{\chi+2 \mu}\right)^{1 / 2}$ characterizes the coupling of linear and rotational motion arising from the micromotion of the fluid molecules or the lubricant additives. Thus $N$ signifies the coupling between the Newtonian and rotational viscosities. As $\chi$ tend to zero, $N$ also tends to zero, and the expressions for the bearing characteristics obtained in this paper reduce to their counter parts in classical Newtonian theory. The second non-dimensional parameter $\bar{l}=\left(\frac{l}{h_{m 0}}\right)$ with $l=\left(\frac{\gamma}{4 \mu}\right)^{1 / 2}$, characterizes an interaction between the bearing geometry and the fluid. In this paper, with the aid of these two non-dimensional parameters, the steady-state performance and dynamic characteristics of one dimensional porous inclined slider bearing is studied. The effect of permeability on the static and dynamic characteristics of the bearings is analyzed through the permeability parameter $\psi\left(=\frac{\phi H}{h_{m_{0}}^{3}}\right)$. In the limiting case

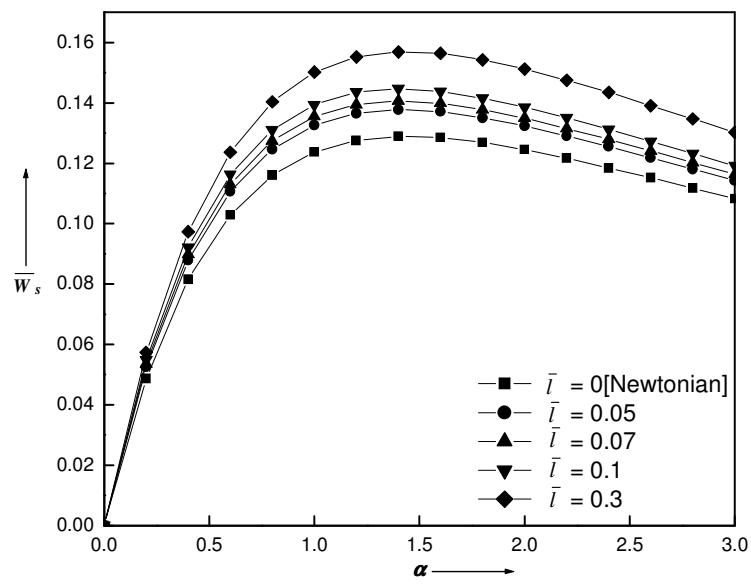

Fig. 2. Variation of nondimensional steady load-carrying capacity $\bar{W}_{s}$ with profile parameter $\alpha$ for $\psi=0.1$ and $N=0.5$.

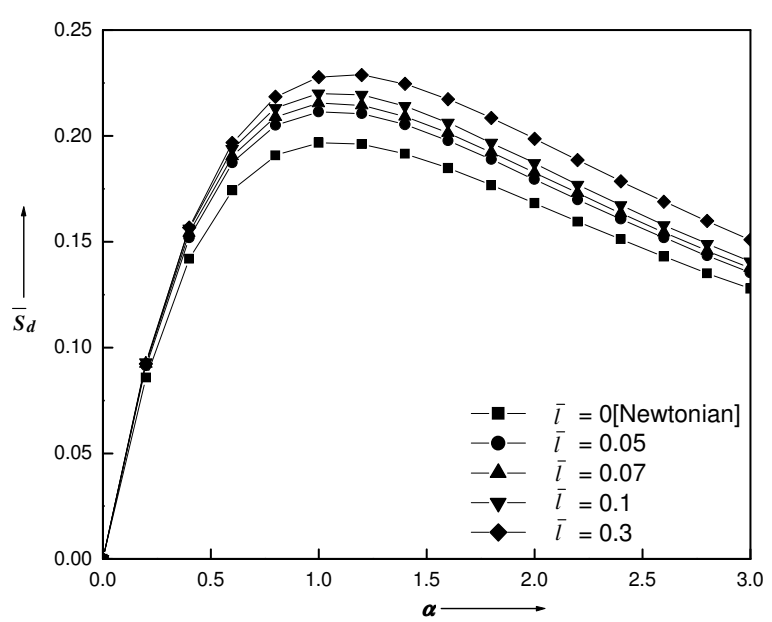

Fig. 3. Variation of nondimensional dynamic stiffness coefficient $\bar{S}_{d}$ with profile parameter $\alpha$ for $\psi=0.1$ and $N=0.5$.

$\psi=0$, the modified Reynolds Eq. (23) reduces to the solid case [11].

Figure 2 presents the variation of non-dimensional steady-load carrying capacity $\bar{W}_{s}$ with profile parameter $\alpha$ for different values of characteristic length $\bar{l}$ with coupling number $N=0.5$ and permeability parameter $\psi=0.1$ It is observed that the effect of $\bar{l}$ is to increase $\bar{W}_{s}$ as compared to the Newtonian case $(\bar{l}=0)$. Further it is also observed the existence of the critical value $\alpha_{c}$ for the profile parameter $\alpha$ at which the steady-load carrying capacity attains maximum. (For $N=0.5$ and $\bar{l}=0.3, \alpha_{c}=1.4$ ). Figure 3 depicts the variation of non-dimensional dynamic stiffness coefficient $\bar{S}_{d}$ with profile parameter $\alpha$ for different values of $\bar{l}$ with $N=0.5$ and $\psi=0.1$. It is observed that the effect of characteristic length of the lubricant is to increase $\bar{S}_{d}$ as compared to the Newtonian case $(\bar{l}=0)$. Further it is observed that the existence of critical 


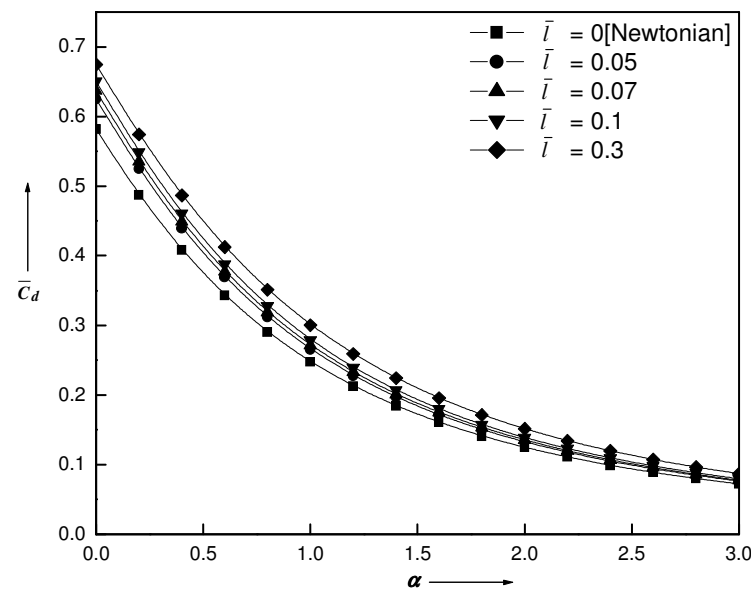

Fig. 4. Variation of nondimensional dynamic damping coefficient $\bar{C}_{d}$ with profile parameter $\alpha$ for $\psi=0.1$ and $N=0.5$.

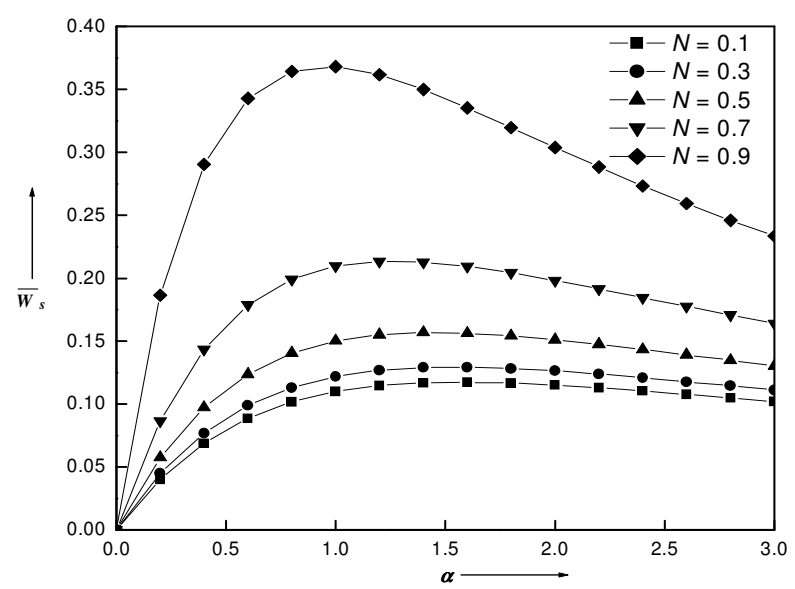

Fig. 5. Variation of nondimensional steady load-carrying capacity $\bar{W}_{s}$ with profile parameter $\alpha$ for $\psi=0.1$ and $\bar{l}=0.3$.

value $\alpha_{c}$ at which $\bar{S}_{d}$ attains the maximum and is found to be independent of $\bar{l}$.

The variation of non-dimensional dynamic damping coefficient $\bar{C}_{d}$ with the profile parameter $\alpha$ for different values of $\bar{l}$ with $N=0.5$ and $\psi=0.1$ is shown in Fig. 4. It is observed that the effect of $\bar{l}$ is to increase $\bar{C}_{d}$ for the higher values of $\alpha$. But there is moderate increase in the value of $\bar{C}_{d}$ for the inclined slider bearing with the higher profile parameter. Further it is observed that $\bar{C}_{d}$ decreases for increasing values of $\alpha$.

Figure 5 depicts the non-dimensional steady-load carrying capacity varies as a function of profile parameter $\alpha$, for different values of $N$, keeping the non-dimensional characteristic length constant, $\bar{l}=0.3$ and $\psi=0.1$. The enhancement of $\bar{W}_{s}$ for higher values the coupling parameter $N$ is pronounced. Because of increase in the viscosity due to the additives, characterized by micropolar fluids, the critical value of the profile parameter $\alpha, \alpha_{c}$ is such value of $\alpha$ at which the steady load

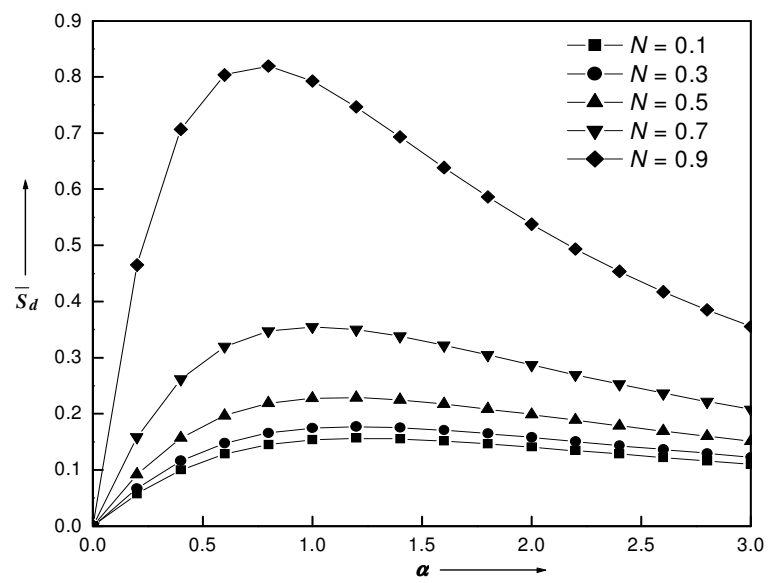

Fig. 6. Variation of nondimensional dynamic stiffness coefficient $\bar{S}_{d}$ with profile parameter $\alpha$ for $\psi=0.1$ and $\bar{l}=0.3$.

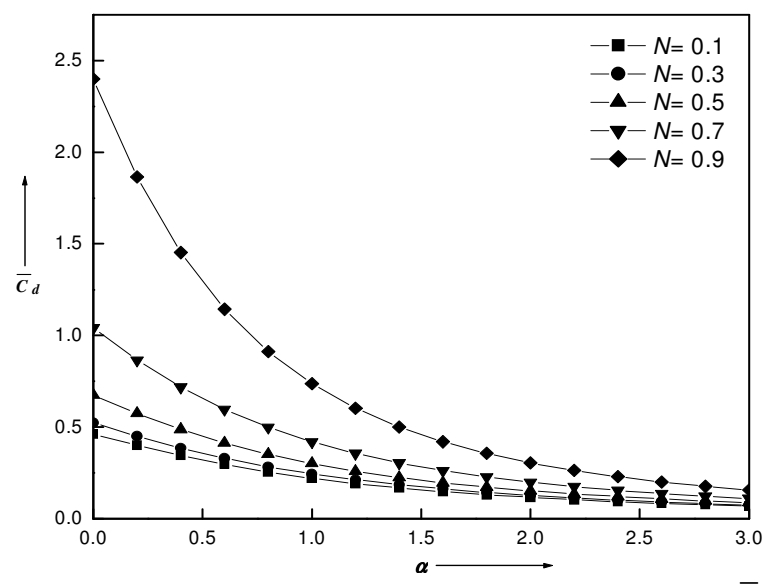

Fig. 7. Variation of nondimensional dynamic damping coefficient $\bar{C}_{d}$ with profile parameter $\alpha$ for $\psi=0.1$ and $\bar{l}=0.3$.

carrying capacity $\bar{W}_{s}$ and the non-dimensional dynamic stiffness coefficient $\bar{S}_{d}$ attains their maximum. It is interesting to note that the critical value $\alpha, \alpha_{c}$ is a function of coupling parameter $N, \alpha_{c}$ decreases for the higher values of $N$.

Figure 6 shows the variation of non-dimensional dynamic stiffness coefficient $\bar{S}_{d}$ with profile parameter $\alpha$ for different values of $N$ with $\bar{l}=0.3$ and $\psi=0.1$. It is observed that, the effect of coupling parameter is to increase the value of $\bar{S}_{d}$. The variation of non-dimensional dynamic damping coefficient $\bar{C}_{d}$ with the profile parameter $\alpha$ with $\bar{l}=0.3$ and $\psi=0.1$ for different values of the coupling number $N$ is shown in Fig. 7. It is observed that the increase in profile parameter $\alpha$ decreases the value of $\bar{C}_{d}$. Further, the $\bar{C}_{d}$ increases for increasing values of $N$. Fig. 8. displays the variation of non-dimensional steady-load carrying capacity $\bar{W}_{s}$ with profile parameter $\alpha$ for different values of permeability parameter $\psi$ with coupling 


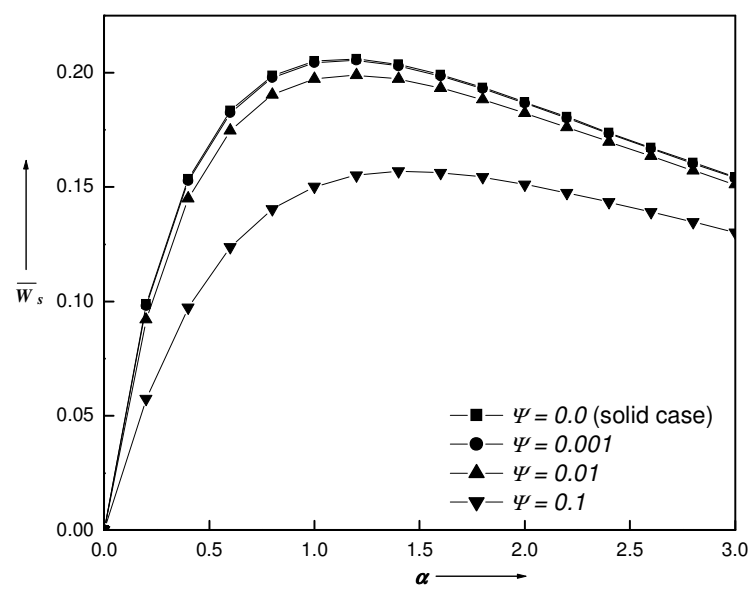

Fig. 8. Variation of nondimensional steady load-carrying capacity $\bar{W}_{s}$ with profile parameter $\alpha$ for $N=0.5$ and $\bar{l}=0.3$.

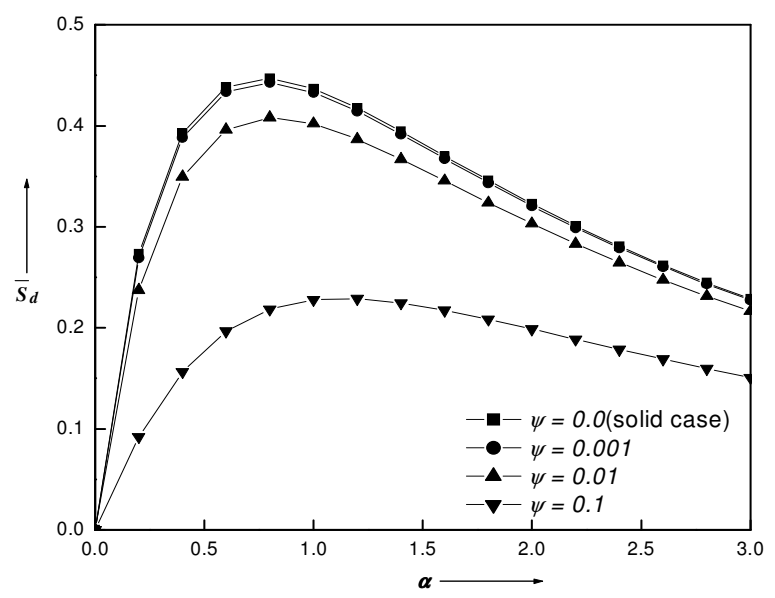

Fig. 9. Variation of nondimensional dynamic stiffness coefficient $\bar{S}_{d}$ with profile parameter $\alpha$ for $N=0.5$ and $\bar{l}=0.3$.

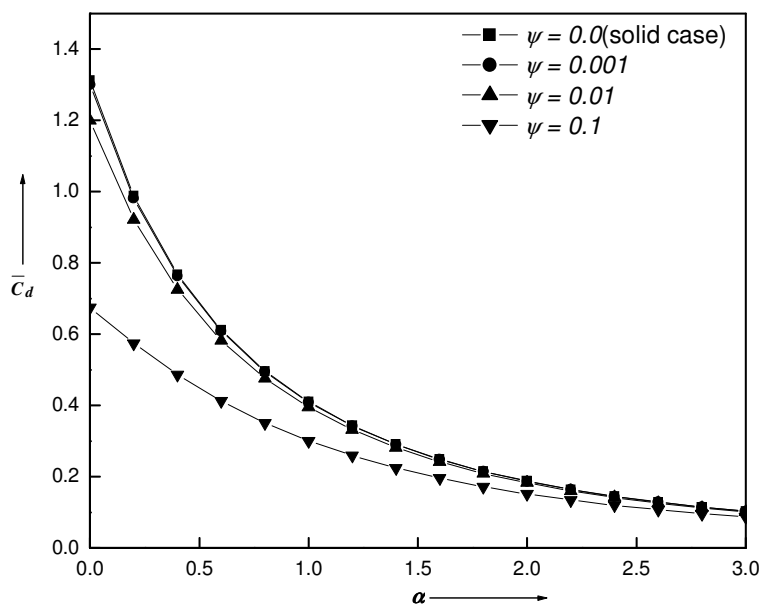

Fig. 10. Variation of nondimensional dynamic damping coefficient $\bar{C}_{d}$ with profile parameter $\alpha$ for $N=0.5$ and $\bar{l}=0.3$.

number $N=0.5$ and characteristic length $\bar{l}=0.3$. It is ob-

served that the effect of $\psi$ is to decrease the value of $\bar{W}_{s}$. When the permeability is very high (larger values of $\psi$ ) the porous material becomes the main path of flow and hence decreases the value of $\bar{W}_{s}$. Further it is also observed that the critical value $\alpha \alpha_{c}$ is a function of the permeability parameter, $\psi$. The value of $\alpha_{c}$ increases for increasing values of $\psi$.

Figure 9 depicts the variation of non-dimensional dynamic stiffness coefficient $\bar{S}_{d}$ with profile parameter $\alpha$ for different values of permeability parameter $\psi$ with $N=0.5$ and $\bar{l}=0.3$. It is observed that as permeability parameter $\psi$ increases, the value of $\bar{S}_{d}$ decreases. The variation of non-dimensional dynamic damping coefficient $\bar{C}_{d}$ with the profile parameter $\alpha$ for different values of permeability parameter $\psi$ for $N=0.5$ and $\bar{l}=0.3$ is shown in Fig. 10. It is observed that the increase of the profile parameter $\alpha$ decreases the value of $\bar{C}_{d}$. The marginal effect of $\psi$ on the variation of $\bar{C}_{d}$ is observed.

\section{CONCLUSIONS}

The dynamic Reynolds-type equation for the porous slider bearings with squeezing effect is derived on the basis of Eringen [6] theory for micropolar fluids. The numerical results are obtained for wide inclined porous slider bearings. On the basis of the results presented; the following conclusions can be drawn.

1. The critical value of the profile parameter $\alpha, \alpha_{c}$ exists such that the steady-state load carrying capacity $\bar{W}_{s}$ and the dynamic stiffness coefficient $\bar{S}_{d}$ attains the maximum value.

2. The critical value of the profile parameter $\alpha_{c}$ is a function of $N$ and $\psi$.

3. The presence of the porous facing on the slider, decreases the $\bar{W}_{s}, \bar{S}_{d}, \bar{C}_{d}$.

4. The micropolar fluid lubricants provide an increased steady-load carrying capacity and dynamic stiffness coefficient whereas decreases the dynamic damping coefficient.

5. The adverse effects due to the presence of porous facing on the slider can be compensated by the proper choice of lubricants with appropriate additives.

\section{ACKNOWLEDGEMENT}

The authors sincerely acknowledge the financial assistance by the UGC, New Delhi, India under the major research project No.F.No.31-84/2005 (SR) and one of the authors (GBM) thankful to Dr. Ashok S. Shettar, Principal, B.V.B. College of Engineering and Technology, Hubli-31 for his encouragements.

\section{NOMENCLATURE}

$a$

$B$

$C_{d}$ difference between the inlet and outlet film thickness width of the bearing dynamic damping coefficient 
$\bar{C}_{d} \quad$ non-dimensional dynamic damping coeffcient

$$
\left(=\frac{C_{d} h_{m 0}^{2}}{\mu U L^{2}}\right)
$$

$h \quad$ film thickness function

$h_{m} \quad$ minimum film thickness at the outlet

$h_{s} \quad$ slider profile function

$h_{m 0} \quad$ steady-state minimum film thickness at the outlet

$H$

$i$

$l \quad$ characteristic material length $\left(=\frac{\gamma}{4 \mu}\right)^{1 / 2}$

$\bar{l} \quad$ non-dimensional characteristic material length

$$
\left(=\frac{l}{h_{m 0}}\right)
$$

$N \quad$ coupling number $\left(=\frac{\chi}{\chi+2 \mu}\right)^{1 / 2}$

$L \quad$ length of the bearing

$p \quad$ dynamic film pressure

non-dimensional dynamic film pressure $\left(=\frac{p h_{m 0}^{2}}{\mu U L}\right)$

$p^{*} \quad$ pressure in the porous region

$p_{0} \quad$ steady film pressure

$\bar{p}_{0} \quad$ non-dimensional steady film pressure $\left(=\frac{p_{0} h_{m 0}^{2}}{\mu U L}\right)$

$p_{1} \quad$ perturbed film pressure

$\bar{p}_{1} \quad$ non-dimensional perturbed film pressure $\left(=\frac{p_{1} h_{m 0}^{2}}{\mu U L}\right)$

$S_{d} \quad$ dynamic stiffness coefficient,

$\bar{S}_{d} \quad$ non-dimensional dynamic stiffness coefficient

$\left(=\frac{S_{d} h_{m 0}^{2}}{\mu U L^{2}}\right)$

$t \quad$ time

$u, v, w \quad$ velocity components

$U \quad$ sliding velocity of the lower part

$W_{s} \quad$ steady load carrying capacity,

$\bar{W}_{s} \quad$ non-dimensional steady load carrying

capacity $\left(=\frac{W_{s} h_{m 0}}{\mu U L^{2}}\right)$

$W_{d} \quad$ perturbed film force,

$\bar{W}_{d} \quad$ non-dimensional perturbed film force $\left(=\frac{W_{d} h_{m 0}}{\mu U L^{2}}\right)$

$v_{1}, v_{2}, v_{3}$ microrotational velocities

$x, y, z \quad$ Cartesian rectangular coordinates $\alpha$

\section{$\delta$}

$\varepsilon$

$\gamma, \chi \quad$ viscosity coefficients for micropolar fluids

$\mu \quad$ classical viscosity coefficient

$\phi \quad$ permeability of the porous material

$\psi \quad$ permeability parameter $\left(=\frac{H \phi}{h_{m 0}^{3}}\right)$

\section{REFERENCES}

1. Agrawal, V. K., Ganju, K. L. and Jethi, S. C., "Squeeze film and externally pressurized bearings micropolar fluid lubricated," Wear, Vol. 19, pp. 259-265 (1972).

2. Balaram, M., "Micropolar squeeze film," ASME Journal of Lubrication Technology, Vol. 97, No. 4, pp. 635-637 (1975).

3. Bujurke, N. M., Bhavi, S. G. and Hiremath, P. S., "Squeeze film lubricated with micropolar Fluids," Proceedings of Indian National Science Academy, Vol. 53, No. 3, pp. 391-398 (1987).

4. Chandan Singh. and Prawal Sihna., "The three-dimensional Reynolds equation for micropolar fluid lubricated bearings," Wear, Vol. 76, pp. 199-209 (1982).

5. Eringen, A. C., "Simple microfluids," International Journal of Engineering Science, Vol. 2, pp. 205-217 (1964).

6. Eringen, A. C., "Theory of micropolar fluids," Journal of Mathematics and Mechanics, Vol. 16, No.1, pp.1-18 (1966).

7. Huang, T. W., "Analysis of finite width journal bearings with micropolar fluids," Wear, Vol. 123, pp. 1-12 (1988).

8. Khonasari, M. M., and Brewe, D. E., "On the performance of finite journal bearings lubricated with micropolar fluids," Tribology Transactions, Vol. 32, No. 2, pp. 155-160 (1989).

9. Kumar, V., "Friction of a plane porous slider of optimal profile," Wear, Vol. 62, pp. 417-418 (1980).

10. Murti, P. R. K., "Analysis of porous slider bearings," Wear, Vol. 28, pp.131-134 (1974).

11. Naduvinamani, N. B. and Marali, G. B., "Dynamic Reynolds equation for micropolar fluids and analysis of the plane slider bearings with squeezing effect," Proceedings of Institution of Mechanical Engineers., Part J: Journal of Engineering Tribology, Vol. 221, J7, pp. 823-829 (2007).

12. Pinkus, O. and Sternlicht, B., Theory of Hydrodynamic Lubrication, McGraw-Hill, New York (1961).

13. Prawal, S., "Dynamically loaded micropolar fluid lubricated journal bearings with special reference to squeeze films under fluctuating loads," Wear, Vol. 45, pp. 279-292 (1977).

14. Ramanaihah, G. and Dubey, J. N., "Optimum slider profile of a slider bearing lubricated with a micropolar fluid," Wear, Vol. 42, pp. 1-7 (1977)

15. Sanni, S. A. and Ayomidele, I. O., "Hydrodynamic lubrication of a porous slider: limitation of the simplifying assumption of a small porous facing thickness," Wear, Vol. 147, pp. 1-7 (1991).

16. Srinivasan, U., "The analysis of double layered porous slider bearings," Wear, Vol. 42, pp. 205-215 (1977).

17. Shukla , J. B. and Isa, M., "Generalized Reynolds equation for micropolar lubricants and its application to one dimensional slider bearing: effects of solid particle additives in solution," Journal of Mechanical Engineering Science, Vol. 17, No. 5, pp. 280-284 (1975).

18. Verma, P. D. S., Agrawal, V. K., and Bhatt, S. B., "The optimum profile for a porous slider bearing," Wear, Vol. 48, pp. 9-14 (1978).

19. Verma, P. D. S., Agrawal, V. K., and Bhatt, S. B., "Porous inclined slider bearing lubricated with micropolar fluid," Wear, Vol. 53, pp. 101-106 (1979).

20. Zaheeruddin, K. H. and Isa, M., "Micropolar fluid lubrication of 
one-dimensional journal bearing," Wear, Vol. 50, pp. 211-220 (1978).

21. Zaheeraddin, K. H. and Isa, M., "Characteristic of a micropolar lubricant in a squeeze film porous spherical bearing," Wear, Vol. 51, pp. 1-10
(1978).

22. Zaheeruddin, K. H. and Isa, M., "One-dimensional porous journal bearing lubrication with micropolar fluid," Wear, Vol. 83 , pp. 257 (1980). 